\title{
Características estructurales de los bosques de Austrocedrus chilensis afectados por "mal del ciprés" en Patagonia, Argentina
}

\author{
Stand structure of Austrocedrus chilensis forests affected by "mal del ciprés" \\ "Andean cypress disease" in Patagonia, Argentina
}

\author{
Ludmila La Manna, ${ }^{1 *}$, José Bava1, Marta Collantes $^{2,3}$ y Mario Rajchenberg, 2 \\ * Autor de correspondencia: ${ }^{1}$ Centro de Investigación y Extensión Forestal Andino Patagónico (CIEFAP), CC 14 (9200), \\ Esquel, Chubut, Argentina, Tel/Fax: 54 (2945) 453948 / 450175, ludmila@ ciefap.org.ar. \\ ${ }^{2}$ Consejo Nacional de Investigaciones Científicas y Técnicas (CONICET), Argentina. \\ ${ }^{3}$ Museo Argentino de Ciencias Naturales Bernardino Rivadavia, Argentina.
}

\begin{abstract}
SUMMARY
Spatial distribution of Austrocedrus chilensis forests includes an area of high climatic, topographic and edaphic heterogeneity. These forests suffer a widespread mortality, locally known as "Andean cypress disease" (mal del ciprés). In this study the stand structure of A. chilensis forests affected by the disease was characterized, covering a range of soils. Three types of stand structure were found: (a) stands with dominance of trees with diameter at $1.30 \mathrm{~m}$ height (DBH) lower than $15 \mathrm{~cm}$, with low values for basal area and height in dominant trees, associated to non volcanic or non allophanized soils; (b) stands with predominance of trees with DBH from 5 to $35 \mathrm{~cm}$, with intermediate values for basal area and height in dominant trees, associated to allophanized volcanic soils; (c) stands with predominance of trees with DBH from 5 to $45 \mathrm{~cm}$ and with gross trees which survived old fires, with the greatest values for basal area and height in dominant trees, associated to sites near streams presenting alluvial or volcanic soils. "Andean cypress disease" was detected in the three stand structure types, suggesting that the disease is not restricted to a particular structure.
\end{abstract}

Key words: Cypress, decline, soil, stand structure.

\section{RESUMEN}

La distribución de los bosques de Austrocedrus chilensis incluye un área de alta variabilidad climática, topográfica y edáfica. Estos bosques sufren un proceso de mortalidad masiva conocido como "mal del ciprés". En este estudio se caracterizó la estructura de los bosques de A. chilensis afectados por "mal del ciprés" en distintos tipos de suelo. Se evidenciaron tres tipos de estructuras de bosque: (a) rodales con dominancia de árboles de diámetro a 1,3 m de altura (DAP) menor a $15 \mathrm{~cm}$, con bajos valores de área basal y altura dominante, asociados a suelos no volcánicos o volcánicos no alofanizados; (b) rodales con predominio de los árboles de 5 hasta $35 \mathrm{~cm}$ de DAP, con valores intermedios de área basal y altura dominante, asociados a suelos volcánicos alofanizados; (c) rodales con predominio de los árboles de 5 hasta $45 \mathrm{~cm}$ de DAP y con árboles remanentes de gran porte, con valores máximos de área basal y altura dominante, asociados a sitios aledaños a cursos de agua con suelos aluviales o volcánicos. El "mal del ciprés" estuvo presente en los distintos tipos de estructura del bosque, evidenciando que la enfermedad no está restringida a una estructura en particular.

Palabras clave: ciprés de la cordillera, declinación, estructura, suelo.

\section{INTRODUCCIÓN}

El ciprés de la cordillera (Austrocedrus chilensis [D.Don] Pic. Serm. et Bizarri) es una Cupressaceae endémica de los bosques andino patagónicos de alto valor maderero (Díaz-Vaz 1985) y paisajístico. En la vertiente occidental de Los Andes, en Chile, A. chilensis se extiende hacia el norte formando poblaciones aisladas hasta los 32³9'S (Schlegel 1962), y en forma más continua entre los $34^{\circ} 45^{\prime}$ y $38^{\circ} \mathrm{S}$. También aparece esporádicamente entre los $42^{\circ} \mathrm{S}$ y $44^{\circ} \mathrm{S}$ (Veblen et al.1995) y en la Cordillera de la Costa (Veblen y Schlegel 1982). Los bosques de A. chilensis presentan mayor desarrollo en la vertiente oriental de Los Andes, en Argentina, cubriendo cerca de 135.000 ha (Bran et al. 2002). Estos bosques se ubican en el piedemonte cordillerano entre los $36^{\circ} 30^{\prime}$ y los $39^{\circ} 30^{\prime} \mathrm{S}$ en forma discontinua y, en forma más continua entre los $39^{\circ} 30^{\prime}$ y los $43^{\circ} 35^{\prime} \mathrm{S}$, en una franja de hasta $60-80 \mathrm{~km}$ de ancho, que incluye un fuerte gradiente pluviométrico. Hacia el oeste, en sitios con precipitaciones entre 1.600 y 
$2.000 \mathrm{~mm}$, forma bosques mixtos con especies del género Nothofagus (Dezzotti y Sancholuz 1991). En áreas con precipitaciones anuales entre 600 y $1.600 \mathrm{~mm} \mathrm{~A}$. chilensis forma bosques monoespecíficos de distintas densidades. Con la disminución de la precipitación hacia el este, el bosque se torna cada vez más abierto, primero en forma de bosquetes aislados en el ecotono bosqueestepa y, finalmente, con individuos aislados en una matriz de estepa (Veblen et al. 1996).

Los bosques de A. chilensis pueden ser de estructura coetánea o disetánea. En sitios húmedos A. chilensis sigue un modo de regeneración catastrófico en respuesta al fuego (Veblen et al. 1995). Es decir, la población se establece en un período de tiempo relativamente corto en respuesta a un disturbio devastador de un rodal y la repentina disponibilidad de recursos (Veblen 1992). Así se establece una regeneración densa y homogénea, a partir de árboles remanentes, generando una estructura regular, donde todos los árboles tienen edades semejantes. Sin embargo, estos bosques usualmente mantienen una estructura irregular en cuanto a la distribución de frecuencias diamétricas, hasta que los individuos de las clases sociales más bajas son eliminados por competencia (Bava y Gonda 1993). La velocidad de este proceso está determinada por las condiciones de colonización postfuego que dependen, entre otros aspectos, de la disponibilidad de semillas (Raffaele y Gobbi 1996, Urretavizcaya y Defossé 2004), condiciones ambientales (Loguercio 1997) y de micrositio (Gobbi y Sancholuz 1992). En sitios secos, el proceso de establecimiento de la regeneración presenta una dinámica diferente, donde la protección que generan los arbustos y los cipreses establecidos previamente juega un rol fundamental. Se forman así estructuras disetáneas, en cuanto a la distribución de edades, e irregulares en cuanto a la distribución de frecuencias diamétricas (Loguercio et al. 1999). Tanto en sitios húmedos como en sitios secos el proceso de regeneración puede ser interferido o inhibido por la acción del ganado (Loguercio et al. 1999).

Austrocedrus chilensis tiene un crecimiento relativamente rápido, incluso a edades avanzadas, si las condiciones son adecuadas (Schmaltz y Gonda 1991, Bava y Gonda 1993). El crecimiento en diámetro es variable, dependiendo de la densidad y las condiciones del sitio, pudiendo alcanzar valores superiores a $1 \mathrm{~cm} /$ año (Donoso 1993). Los mayores crecimientos, densidad y área basal se registran en los bosques puros y densos desarrollados en sitios con precipitaciones anuales entre 900 y $1.600 \mathrm{~mm}$ (Dezzotti y Sancholuz 1991).

Los bosques de A. chilensis no sólo se desarrollan en un área de alta variabilidad climática, sino también en un ambiente de gran variabilidad geomórfica producto de la acción glacial (Rabassa et al. 2000, Haller 2001). Durante el Holoceno el paisaje fue cubierto por depósitos volcánicos, los cuales constituyen los materiales originarios de la mayoría de los suelos de la región (Apcarian e
Irisarri 1993, Colmet Dâage et al. 1995). Los bosques densos de A. chilensis se desarrollan sobre suelos altamente diversos, desde suelos volcánicos hasta suelos glaciales vérticos (vertisoles) (La Manna 2005). En función de las condiciones de precipitación y drenaje, a partir de la alteración de ceniza volcánica, puede formarse alófano, que es un aluminosilicato amorfo que otorga a los suelos alta retención de agua y fertilidad, determinando características apropiadas para el desarrollo forestal (Colmet Dâage et al. 1995, López 1996). Por el contrario, las propiedades físicas de los suelos vérticos constituyen una gran limitante para el desarrollo de la vegetación (Eswaran et al. 1999) y se han evidenciado fuertes dificultades de enraizamiento en bosques de $A$. chilensis desarrollados sobre este tipo de suelos (La Manna et al. 2004). Es escasa aún la información disponible sobre las características estructurales del bosque de A. chilensis en distintos tipos de suelo (Le-Quesne 1988, Goya et al. 1998). Numerosas especies forestales presentan distinta estructura y capacidad de crecimiento, de acuerdo a las características edáficas del sitio en que se están desarrollando (Pritchett 1986, Schlatter y Gerding 1995, Shaw et al. 2001).

Los bosques de A. chilensis son sometidos a la extracción de madera y leña, a una fuerte presión de herbivoría (Veblen et al. 1995, Relva y Veblen 1998) y a frecuentes incendios, generalmente de origen antrópico (Loguercio et al. 1999). Otra de las amenazas que afecta a los bosques de A. chilensis es la existencia de mortandad masiva de árboles, cuya causa es aún discutida (Havrylenko et al. 1989, Rosso et al. 1994, Greslebin y La Manna 2004) y parece enmarcarse en el concepto de enfermedades de declinación forestal (Manion 1991, Manion y Lachance 1992). Esta mortandad, conocida como "mal del ciprés", se detectó por primera vez hace aproximadamente 60 años, y su extensión e importancia han ido en aumento (Hranilovic 1988, La Manna y Carabelli 2005).

El "mal del ciprés" ha sido asociado con factores bióticos, tales como hongos Aphyllophorales causantes de pudriciones radicales, cuya acción sería secundaria (Barroetaveña y Rajchenberg 1996) y distintas especies de Phytophthora, hongos oomycetes que tienen capacidad patógena a nivel de las raíces primarias (Rajchenberg et al. 1998, Greslebin et al. 2004, 2005). El "mal del ciprés" también ha sido asociado a factores abióticos tales como altas precipitaciones, altitudes medias (Baccalá et al. 1998), terremotos y períodos secos seguidos por períodos húmedos previos al inicio del decaimiento (Calí 1996). Estudios edáficos han evidenciado que existen marcadas diferencias de suelo entre el bosque con árboles enfermos y el bosque sin síntomas, estando la enfermedad asociada a suelos mal drenados (La Manna y Rajchenberg 2004ab).

Sobre la base de los antecedentes descritos se planteó este estudio cuyos objetivos fueron: a) caracterizar la 
estructura de los bosques de A. chilensis en distintos tipos de suelo, y b) evaluar diferencias estructurales entre el bosque con árboles enfermos y el bosque sin síntomas de declinación. Las hipótesis involucradas son que: a) existe una asociación entre el tipo de suelo y la estructura del bosque de A. chilensis de tal manera que habrá menor altura dominante y menor área basal en suelos no volcánicos, y b) existe una asociación entre la estructura y el estado sanitario del bosque de A. chilensis que se aprecia como menor altura dominante y menor área basal en bosques enfermos.

\section{MÉTODOS}

El estudio se llevó a cabo en el SO de la provincia de Río Negro y el NO de la provincia de Chubut, Argentina, cubriendo un rango latitudinal desde $41^{\circ} 53^{\prime}$ a $43^{\circ} 13^{\prime} \mathrm{S}$ y longitudinal de $71^{\circ} 23^{\prime}$ a $71^{\circ} 43^{\prime} \mathrm{O}$. Fueron seleccionados diecinueve sitios con bosque puro de A. chilensis con síntomas de "mal del ciprés", abarcando diferentes situa- ciones de geomorfología y un gradiente de precipitación desde 690 a $1.610 \mathrm{~mm}$ anuales. Se incluyeron cuatro sitios control donde la enfermedad estuvo completamente ausente (figura 1). Todos los sitios de muestreo correspondieron a bosques densos postfuego no intervenidos y, en la mayoría de los casos, sometidos a uso ganadero. En cada sitio de muestreo correspondiente a bosques afectados por el "mal del ciprés" se establecieron dos parcelas de $200 \mathrm{~m}^{2}$ : parcela afectada, en un área del bosque con síntomas severos de la enfermedad (más del $90 \%$ de árboles muertos), y parcela asintomática en un área del bosque sin síntomas. En los bosques control, se estableció una parcela de $200 \mathrm{~m}^{2}$ en una zona representativa de la estructura del bosque (parcela control).

Estructura del bosque. Se registró el diámetro a 1,3 m de altura (DAP) y la posición sociológica (dominante, codominante, suprimido) de todos los árboles con DAP mayor o igual a $5 \mathrm{~cm}$ incluidos en la parcela. Se determinó también la altura de los árboles dominantes, mediante un clinómetro, siguiendo el criterio de los 100 ejemplares
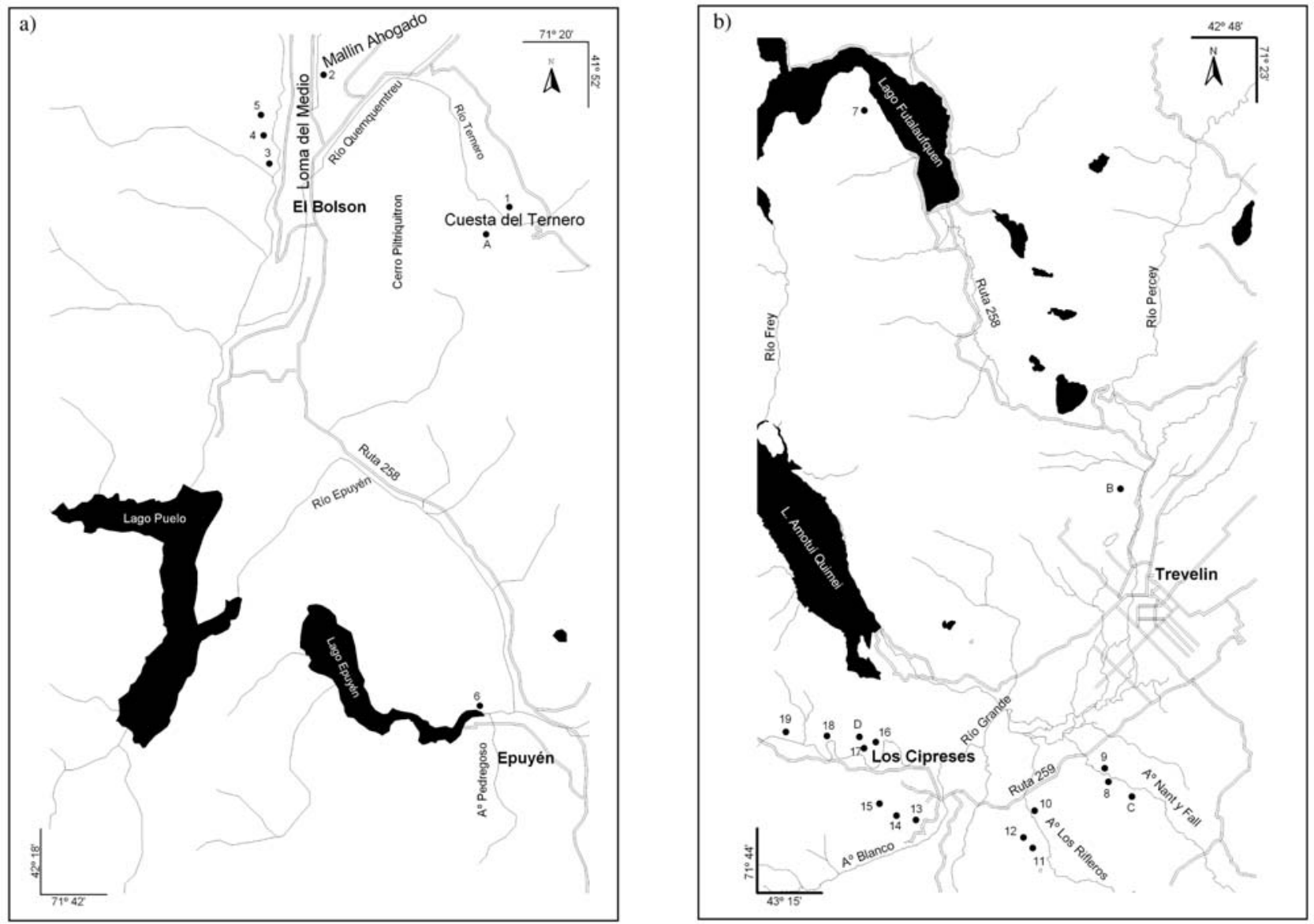

Figura 1. Ubicación de los Sitios de muestreo. a) Área El Bolsón-Epuyén (Río Negro-Chubut, Argentina); b) Área Valle 16 de Octubre (Chubut, Argentina). A, B, C y D corresponden a bosques control, donde la enfermedad está ausente.

Location of Sampling sites. a) Area El Bolsón-Epuyén (Río Negro-Chubut, Argentina). b) Area Valle 16 de Octubre (Chubut, Argentina). A, B, C and D are Control sites, where the disease is totally absent. 
más gruesos por hectárea (Daniel et al. 1982). Con estos valores se obtuvieron los parámetros dasométricos del rodal: número de individuos por hectárea, área basal (AB), frecuencias diamétricas, diámetro cuadrático medio (DCM) y altura dominante. La edad de los árboles dominantes fue estimada mediante el conteo de anillos de muestras obtenidas con el barreno de Pressler o de cuñas de árboles muertos, cuando fue posible. Las muestras de tarugos se obtuvieron de los dos árboles de mayor DAP, por duplicado, a una altura de $50 \mathrm{~cm}$ del suelo.

Los individuos de cada parcela con DAP mayor o igual a $5 \mathrm{~cm}$ fueron clasificados visualmente por clases de vitalidad, siguiendo la escala de Rajchenberg y Cwielong (1993), que establece seis categorías, de 0 (sano) a 5 (muerto). Para caracterizar la condición de declinación de cada parcela se utilizó el porcentaje de AB afectada (\% AB afectada). Este último fue calculado como el porcentaje de $A B$ de árboles enfermos y muertos respecto al $\mathrm{AB}$ total de ciprés. La estructura de las parcelas afectadas fue reconstruida incluyendo en la distribución de frecuencias diamétricas a los árboles muertos.

Caracterización del sitio. La precipitación media anual se obtuvo de los registros de la estación meteorológica más cercana. En cada parcela se determinaron la altitud, el grado de la pendiente y la orientación. La orientación fue descompuesta en dos variables: orientación Sur relativa o grados desde el Norte $(0=$ orientación $\mathrm{N}, 90=$ orientación E u O) y orientación Este relativa o grados desde el Oeste (Anchorena y Cingolani 2002). El perfil del suelo se describió mediante una calicata, siguiendo la metodología propuesta por Schoeneberger et al. (1998). Se caracterizó la profundidad de raíces, el porcentaje de fragmentos gruesos y la abundancia de rasgos redoximórficos (pérdida o ganancia de color originada por procesos de oxidación/ reducción). Estos rasgos, indicadores de drenaje deficiente, fueron detectados visualmente y/o con una solución de $\alpha, \alpha$ '-dipiridilo (Schoeneberger et al. 1998). En el perfil, se tomaron muestras del horizonte A para la determinación de propiedades físicas y químicas: densidad aparente, materia orgánica (Davies 1974), bases de intercambio (Ca, $\mathrm{Mg}, \mathrm{K}$, y Na) (Marbán 2004) y pH en NaF (1:50). El pH en $\mathrm{NaF}$ fue determinado con el objetivo de detectar la presencia de aluminosilicatos amorfos (Fieldes y Perrot 1966); valores superiores a 9,2 unidades indican la presencia de alófano (Irisarri 2000).

Análisis de datos. La estructura del bosque fue descrita y clasificada sobre la base de la distribución de frecuencias diamétricas. Se determinaron los valores medios y desviación estándar de los distintos parámetros para cada tipo de estructura. Las diferencias en los parámetros entre los tipos de estructura fueron evaluadas mediante análisis de varianza y contrastes realizados con el método de Tuckey HSD (honest significant difference) (Ramsey y Schafer 1997). Cuando los datos no cumplieron los supuestos de normalidad y homogeneidad de varianzas se analizaron mediante la prueba de Kruskall-Wallis y se realizaron contrastes de a pares mediante la prueba de comparación de rangos medios (Ramsey y Schafer 1997).

Para distinguir las variables de sitio asociadas con los diferentes tipos de estructura se realizó un análisis discriminante con un método de selección de variables del tipo paso a paso (Afifi y Clark 1984). Cada tipo de estructura hallado fue considerado un grupo y como variables independientes se incluyeron todas las variables de sitio (climáticas, topográficas y edáficas) mencionadas previamente.

Para evaluar las diferencias estructurales entre el bosque enfermo y el bosque sin síntomas de declinación, se realizó una prueba t para muestras apareadas (Sokal y Rohlf 1999).

Para la realización de los análisis se utilizaron los programas SPSS for Windows y Statistix Version 8.0.

\section{RESULTADOS}

Estructura del bosque. De acuerdo a la distribución de frecuencias diamétricas, se identificaron tres tipos de estructuras: (a) con dominancia de árboles de DAP $<15 \mathrm{~cm}$ (clases diamétricas 1 y 2 , figura $2 a$ ); (b) con predominancia de árboles de 5 a $35 \mathrm{~cm}$ de DAP (clases 1 a 6 , figura 2b), y (c) con predominancia de árboles de 5 a 45 $\mathrm{cm}$ de DAP (clases 1 a 8 ) y con árboles remanentes de gran porte (figura $2 \mathrm{c}$ ).

Las distintas distribuciones de frecuencias diamétricas se reflejan en los valores de DCM $[(a)<(b)<(c)]$ (cuadro 1). Las parcelas con estructura tipo (a) presentaron menores valores de AB, DCM, DAP dominante y altura dominante y un alto número de cipreses por hectárea. Las parcelas con estructura tipo (c) presentaron características opuestas: altos valores de AB, DCM, DAP dominante y altura dominante, y un escaso número de árboles por hectárea. El tipo de estructura (b) se caracterizó por presentar valores intermedios de las distintas variables (cuadro 1). Si bien la edad de los árboles dominantes tendió a ser mayor en la estructura (c), los valores fueron altamente variables. Las edades máximas, con alrededor de 170 anillos, fueron registradas en rodales con estructura de tipos (a) y (c) (sitios 9 y 19, respectivamente; figura 1). En tanto las edades mínimas, donde se contabilizaron menos de 60 anillos, fueron registradas en rodales con estructura de tipos (a) y (b) (sitios 1 y 11, respectivamente, figura 1). El valor medio del\% $\mathrm{AB}$ afectada por el "mal del ciprés" fue similar para los distintos tipos de estructura.

Análisis discriminante. $\mathrm{El} \mathrm{pH}$ en $\mathrm{NaF}$ del horizonte A fue identificado como la variable de sitio más fuertemente asociada a los tipos de estructura. Los bosques con estructura del tipo (a) se asociaron a suelos de bajo $\mathrm{pH}$ 

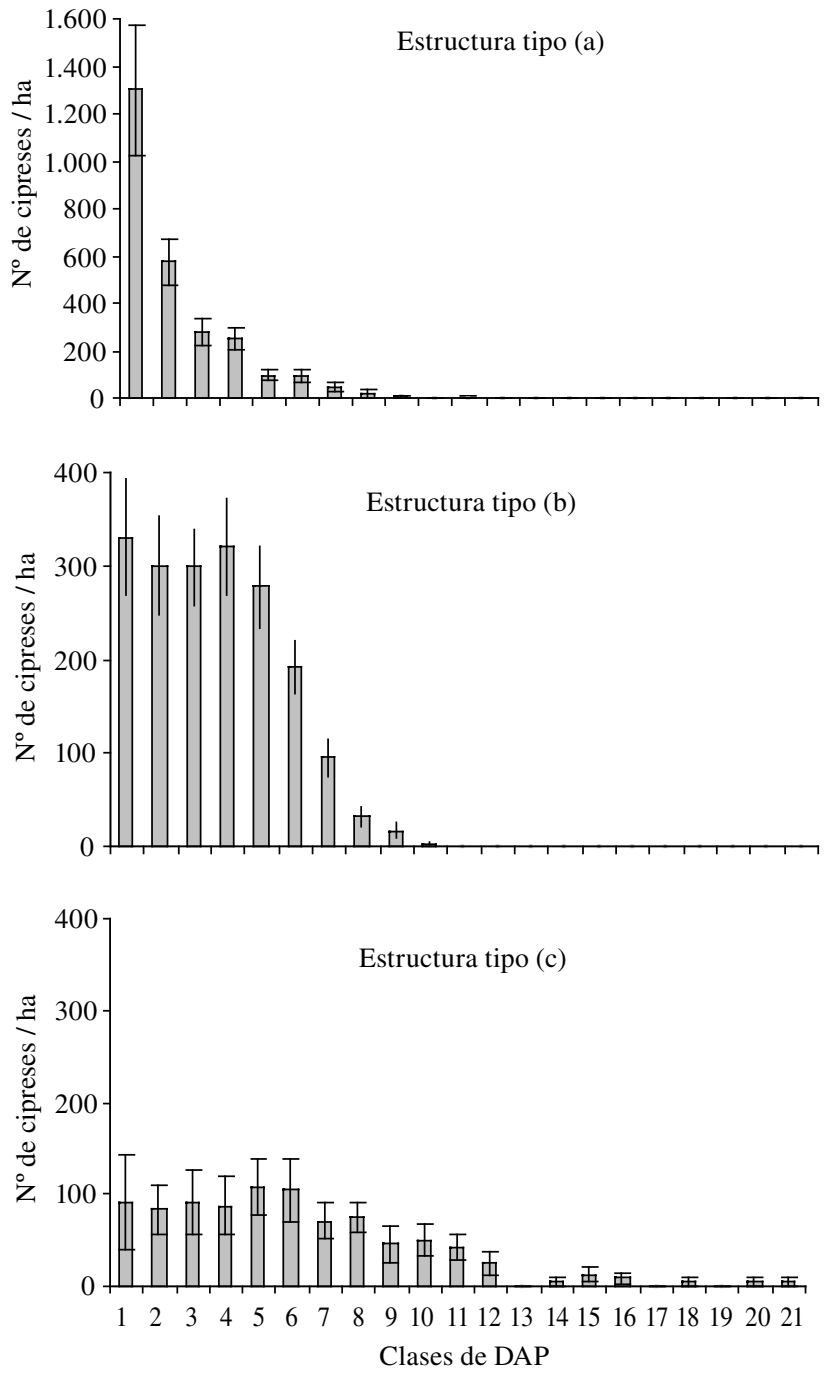

\begin{tabular}{clcl}
\hline DAP & DAP $(\mathrm{cm})$ & DAP & DAP $(\mathrm{cm})$ \\
\hline 1 & {$[5-10)$} & 2 & {$[10-15)$} \\
3 & {$[15-20)$} & 4 & {$[20-25)$} \\
5 & {$[25-30)$} & 6 & {$[30-35)$} \\
7 & {$[35-40)$} & 8 & {$[40-45)$} \\
9 & {$[45-50)$} & 10 & {$[50-55)$} \\
11 & {$[55-60)$} & 12 & {$[60-65)$} \\
13 & {$[65-70)$} & 14 & {$[70-75)$} \\
15 & {$[75-80)$} & 16 & {$[80-85)$} \\
17 & {$[85-90)$} & 18 & {$[90-95)$} \\
19 & {$[95-100)$} & 20 & {$[100-105)$} \\
21 & {$[105-110)$} & & \\
\hline
\end{tabular}

Figura 2. Distribución de frecuencias diamétricas correspondientes a los tres tipos de estructura registrados en bosques puros de A. chilensis. Las clases diamétricas corresponden a intervalos de $5 \mathrm{~cm}$. (Nótese que el gráfico de la Estructura tipo (a) tiene una escala diferente).

Mean diametric distribution of A. chilensis forests corresponding to different stand structure types.
Cuadro 1. Valores medios y error estándar de los parámetros dasométricos correspondientes a cada tipo de estructura registrado en bosques de $A$. chilensis. Letras diferentes indican diferencias significativas $(P<0,05)$.

Mean and standard error of stand parameters corresponding to different stand structure types. Different letters indicate significant differences $(P<0.05)$.

\begin{tabular}{cccr}
\hline Parámetro & Estructura (a) & Estructura (b) & Estructura (c) \\
\hline $\mathrm{N}^{0}$ cipreses / ha & $2.677 \pm 344 \mathrm{a}$ & $1.865 \pm 190 \mathrm{~b}$ & $908 \pm 140 \mathrm{c}$ \\
Área basal (m²/ha) & $55,2 \pm 5,1 \mathrm{a}$ & $76,6 \pm 7,1 \mathrm{~b}$ & $100,2 \pm 6,8 \mathrm{c}$ \\
$\mathrm{DCM}(\mathrm{cm})$ & $17,0 \pm 1,2 \mathrm{a}$ & $23,5 \pm 0,9 \mathrm{~b}$ & $40,9 \pm 3,3 \mathrm{c}$ \\
DAP dominante $(\mathrm{cm})$ & $42,4 \pm 5,3 \mathrm{a}$ & $41,2 \pm 1,4 \mathrm{a}$ & $74,1 \pm 5,1 \mathrm{~b}$ \\
H dominante (m) & $15,7 \pm 1,2 \mathrm{a}$ & $18,6 \pm 0,7 \mathrm{~b}$ & $22,1 \pm 1,0 \mathrm{c}$ \\
Número de anillos & $97,3 \pm 12,0 \mathrm{ab}$ & $86,7 \pm 2,9 \mathrm{a}$ & $123,3 \pm 9,8 \mathrm{~b}$ \\
\% AB afectada & $44,3 \pm 12,4 \mathrm{a}$ & $50,4 \pm 11,0 \mathrm{a}$ & $42,8 \pm 14,2 \mathrm{a}$ \\
por "mal del ciprés" & & & \\
\hline
\end{tabular}

en $\mathrm{NaF}(8,35 \pm 0,25)$, correspondientes a suelos no volcánicos o suelos volcánicos no alofanizados (Irisarri 2000). Por el contrario, los bosques con estructura de tipos (b) y (c) se asociaron a suelos de alto $\mathrm{pH}$ en $\mathrm{NaF}$, es decir, suelos volcánicos alofanizados, con valores medios de 9,80 $\pm 0,20$ y 9,39 $\pm 0,27$, respectivamente. El modelo logrado fue bueno (Wilks's $\lambda=0,700, P<0,001$ ), sin embargo, la función discriminante hallada sólo distinguió eficientemente la estructura (a) de la (b) y la (c) $(P<0,05)$, pero no fue capaz de discriminar la estructura (b) de la (c) $(P>0,05)$. El $83 \%$ de las parcelas con estructura (a) y el 78\% de las parcelas con estructura (b) fueron correctamente clasificados, en tanto sólo el $33 \%$ de las parcelas (c) fue bien clasificado. Los sitios con estructura (c), que evidenciaron un rango amplio de valores de $\mathrm{pH}$ en $\mathrm{NaF}$, presentaron una posición topográfica particular: el $75 \%$ de las parcelas se ubicó cercano a cursos de agua (figura 3 ).

Las correlaciones entre las variables independientes y la función discriminante, incluyendo aquellas variables no incluidas en la función (cuadro 2), indican que el $\mathrm{pH}$ en $\mathrm{NaF}$, los fragmentos gruesos y el contenido de materia orgánica resultaron positivamente relacionados con la función discriminante, mientras que las bases de intercambio ( $\mathrm{K}, \mathrm{Na}, \mathrm{Ca}, \mathrm{Mg})$, la densidad aparente del horizonte A y los rasgos redoximórficos estuvieron negativamente asociados. Esto sugiere que la estructura (a), negativamente relacionada con la función, está asociada a suelos no alofanizados, pobremente drenados, ricos en bases y de baja porosidad. A su vez, la estructura (b) se asoció a suelos volcánicos, ricos en materia orgánica y de baja densidad aparente.

Se observó una asociación entre el tipo de estructura y el material original de los suelos. La mayoría de las parcelas con estructura de tipo (a) correspondieron a suelos con influencias de materiales finos, ya sea por un 


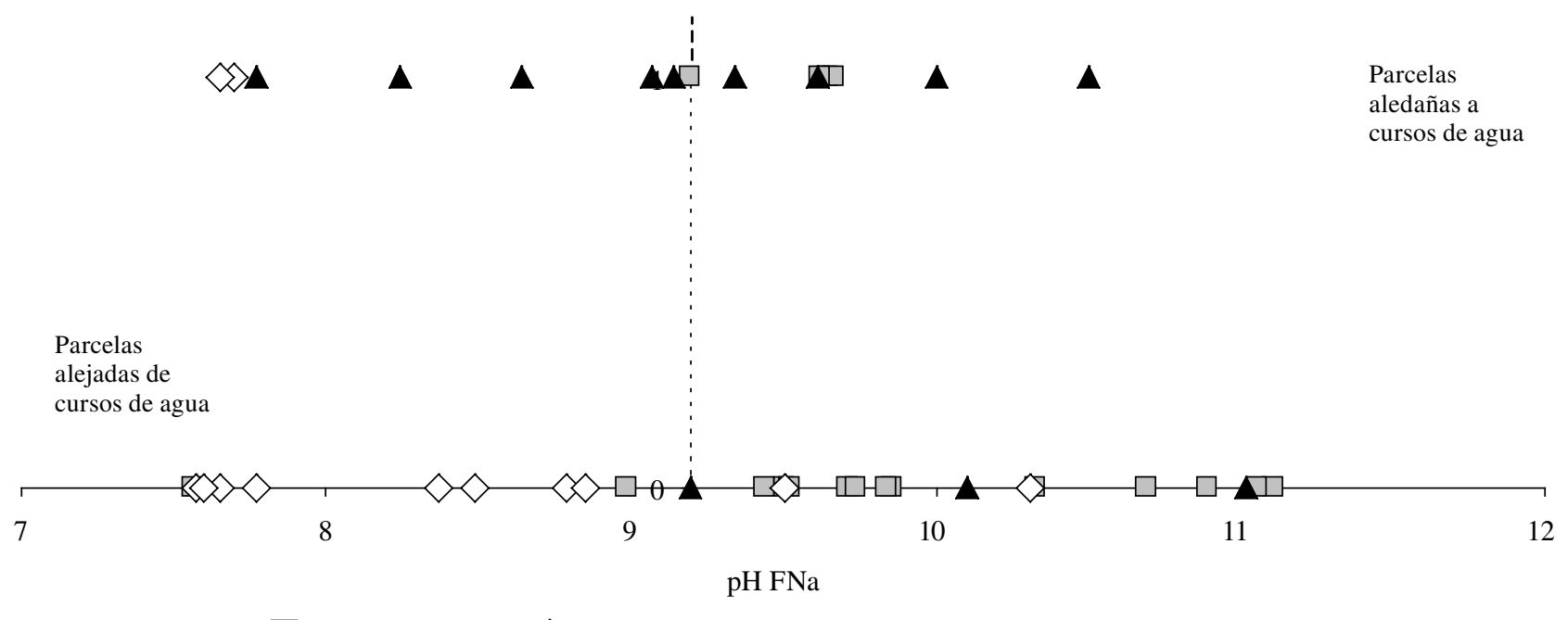

Estructura (a) $\square$ Estructura (b) $\quad \Delta$ Estructura (c)

Figura 3. Valores de $\mathrm{pH}$ en $\mathrm{NaF}$ y ubicación respecto a cursos de agua de parcelas correspondientes a los distintos tipos de estructura registrados en bosques de A. chilensis. La línea punteada indica un valor de $\mathrm{pH}$ en $\mathrm{NaF}$ de 9,2 ; $\mathrm{pH}$ mayores a este valor indican la presencia de alófano (Irisarri 2000).

$\mathrm{pH}$ in $\mathrm{NaF}$ values and distance from streams (upper line: close to streams; lower line: far from streams) of A. chilensis plots corresponding to different stand structure types. The dotted line indicates $9 .-2 \mathrm{pH}$ in $\mathrm{NaF}$; $\mathrm{pH}$ greater than this value suggests the presence of allophane (Irisarri 2000).

Cuadro 2. Coeficientes de correlación entre las variables de sitio y la función discriminante estandarizada.

Correlation coefficients between site variables and the standardized discriminative function.

\begin{tabular}{lc}
\hline Variable & Coeficiente de correlación \\
\hline $\mathrm{pH} \mathrm{FNa}$ & $1,000^{*}$ \\
$\mathrm{~K}$ & $-0,580^{*}$ \\
$\mathrm{Na}$ & $-0,515^{*}$ \\
$\mathrm{Ca}$ & $-0,507^{*}$ \\
Fragmentos gruesos & $0,380^{*}$ \\
Densidad aparente & $-0,365^{*}$ \\
Materia orgánica & $0,338^{*}$ \\
Mg & $-0,327^{*}$ \\
Rasgos redoximórficos & $-0,302^{*}$ \\
Precipitación & 0,221 \\
Profundidad efectiva & 0,128 \\
Pendiente & $-0,072$ \\
Altitud & 0,068 \\
Orientación sur relativa & 0,046 \\
Orientación este relativa & $-0,037$ \\
\hline
\end{tabular}

$* P<0,05$

material original glacial de textura fina (sitios 1 y 9 ) o por un depósito somero de ceniza sobre material fino (sitios 4, 10 y 13; figura 1) (figura 4, cuadro 3). El tipo de estructura (b) correspondió principalmente a parcelas con suelos volcánicos (de ceniza o pumita) con presencia de fragmentos gruesos de diversos tamaños $(2 \mathrm{a} 60 \mathrm{~cm})$ (sitios $7,11,14$ y 15), o bien, suelos con un gran espesor de ceniza $(>100 \mathrm{~cm}$ ) depositada sobre material de texturas finas (sitios 2, 5 y 12; figura 1) (figura 4, cuadro 3). Las parcelas con estructura de tipo (c) se asociaron, en su mayoría, a suelos aluviales con aporte de ceniza volcánica (sitios 6, 8, 16, 18 y 19) y a suelos volcánicos profundos que correspondieron a las parcelas control (sitios A, B, C y D; figura 1) (figura 4, cuadro 3).

Estructura de los bosques enfermos. Como se mencionó anteriormente, los distintos tipos de estructura presentaron similares valores de\% AB afectada (cuadro 1). En la mayoría de los casos, las parcelas asintomática y enferma de un mismo sitio de muestreo correspondieron al mismo tipo de estructura y no se hallaron diferencias en los parámetros dasométricos del rodal al comparar el bosque enfermo y el bosque sin síntomas de declinación (cuadro 4). Sin embargo, es interesante destacar que los cuatro bosques control (sitios A, B, C y D), donde la enfermedad estuvo completamente ausente, correspondieron al tipo de estructura (c).

\section{DISCUSIÓN}

En este trabajo pudieron identificarse distintos tipos de estructuras: rodales con un rango acotado de DAP, con predominancia o no de los árboles de diámetros más pequeños, y rodales con un amplio rango de DAP que 
Cuadro 3. Perfiles de suelo representativos para las distintas estructuras del bosque de A. chilensis.

Representative soil profiles for different $A$. chilensis forest structures.

\begin{tabular}{|c|c|c|c|c|c|c|c|c|}
\hline Horizonte & Profundidad $(\mathrm{cm})$ & Color $^{\mathrm{a}}$ & Textura ${ }^{b}$ & Estructura $^{\mathrm{c}}$ & Raíces $^{\mathrm{d}}$ & $\begin{array}{l}\text { Fragmentos } \\
\text { gruesos }(\%)\end{array}$ & $\begin{array}{l}\text { Rasgos } \\
\text { redox }^{\mathrm{e}}\end{array}$ & Límite $^{\mathrm{f}}$ \\
\hline \multicolumn{9}{|c|}{ Bosques con estructura tipo (a). } \\
\hline \multicolumn{9}{|c|}{ Material original: Depósito glacifluvial de texturas finas } \\
\hline A & $0-15$ & 10YR 3/2 & $\mathrm{Fa}$ & 3 GR F & $2 \mathrm{~F}$ & 0 & - & G W \\
\hline $\mathrm{C} 1$ & $15-40$ & $10 Y R 4 / 2$ & a & 3 WEG M & $1 \mathrm{~F}$ & 1 & $\mathrm{~m} 2 \mathrm{P}$ & G S \\
\hline $\mathrm{C} 2$ & $40-80+$ & $7,5 \mathrm{YR} 5 / 3$ & $\mathrm{a}$ & 3 WEG M & $1 \mathrm{~F}$ & 1 & $\mathrm{~m} 2 \mathrm{P}$ & - \\
\hline \multicolumn{9}{|c|}{ Material original: Ceniza volcánica sobre depósito glacifluvial de texturas finas } \\
\hline Oi & $0-2,5$ & - & - & - & - & - & - & - \\
\hline A & $2,5-13$ & 10YR 3/1 & $\mathrm{F}$ & 3 GR M & $3 \mathrm{~F}$ & 0 & - & $\mathrm{CW}$ \\
\hline $\mathrm{AC}$ & $13-35$ & 10YR 3/2 & $\mathrm{Fl}$ & $1 \mathrm{SBK} M$ & $2 \mathrm{M}$ & 0 & - & G W \\
\hline $\mathrm{C} 1$ & $35-50$ & 10YR 4/1 & $\mathrm{Fa}$ & 2 SBK M & $1 \mathrm{C}$ & 0 & - & $\mathrm{CW}$ \\
\hline $2 \mathrm{C} 2$ & $50-80+$ & $10 Y R 5 / 3$ & $\mathrm{a}$ & $1 \mathrm{SBK}$ CO & $1 \mathrm{M}$ & 0 & m $4 \mathrm{D}$ & - \\
\hline
\end{tabular}

Material original: Ceniza volcánica sobre material rocoso

Bosques con estructura tipo (b)

$\begin{array}{ccccccccc}\text { Oi } & 0-3 & - & - & - & - & - & - & - \\ \text { A } & 3-16 & 10 Y R 2 / 1 & \text { FA } & 2 \text { GR M } & 2 \text { M } & 2 & - & \text { C W } \\ \text { AC } & 16-36 & 10 Y R 3 / 2 & \text { FA } & 1 \text { SBK F } & 3 \text { C } & 10 & - & \text { G I } \\ \text { C1 } & 36-48 & 10 Y R 3 / 3 & \text { FA CNX } & 2 \text { SBK F } & 2 \text { F } & 80 & - & \text { G I } \\ 2 \mathrm{C} 2 & 48-80+ & - & \text { PST } & - & - & 90 & - & -\end{array}$

Material original: Ceniza volcánica sobre depósito glacial de texturas finas

\begin{tabular}{|c|c|c|c|c|c|c|c|c|}
\hline Oi & $0-3$ & - & - & - & - & - & - & - \\
\hline $\mathrm{Oe}$ & $3-5$ & - & - & - & - & - & - & - \\
\hline A1 & $5-17$ & $10 Y R 3 / 2$ & $\mathrm{AF}$ & $1 \mathrm{GR} F$ & $3 \mathrm{M}$ & 0 & - & $\mathrm{C} \mathrm{S}$ \\
\hline $\mathrm{A} 2$ & $17-35$ & $10 Y R 3 / 3$ & $\mathrm{AF}$ & 2 GR F & $3 \mathrm{C}$ & 0 & - & $\mathrm{C} \mathrm{S}$ \\
\hline $\mathrm{ABw}$ & $35-69$ & 10YR 4/4 & $\mathrm{F}$ & $1 \mathrm{SBK} M$ & $3 \mathrm{VC}$ & 0 & - & $\mathrm{C} \mathrm{S}$ \\
\hline $\mathrm{Bw}$ & $69-105$ & 10YR 5/6 & $\mathrm{Fl}$ & $1 \mathrm{SBK} M$ & $1 \mathrm{~F}$ & 0 & - & A S \\
\hline $2 \mathrm{C} 1$ & $105-120$ & 10YR 5/4 & $\mathrm{aA}$ & $1 \mathrm{SBK} M$ & $1 \mathrm{~F}$ & 2 & f $1 \mathrm{D}$ & A S \\
\hline $2 \mathrm{C} 2$ & $120-140+$ & $2,5 Y 5 / 3$ & $\mathrm{aA}$ & $1 \mathrm{SBK} F$ & - & 5 & c $2 \mathrm{P}$ & - \\
\hline
\end{tabular}

Bosques con estructura tipo (c)

Material original: Depósito aluvial con aporte de ceniza volcánica

\begin{tabular}{|c|c|c|c|c|c|c|c|}
\hline Oi & $0-0,5$ & - & - & - & - & - & - \\
\hline A & $0,5-16$ & $10 Y R 3 / 2$ & FA & 0 SGR & $3 \mathrm{~F}$ & c & - \\
\hline $\mathrm{AC}$ & $16-45$ & $10 Y R$ 3/4 & $\mathrm{AF}$ & 0 SGR & $3 \mathrm{C}$ & c & - \\
\hline $\mathrm{C} 1$ & $45-75$ & $10 \mathrm{YR} 4 / 4$ & A & $0 \mathrm{MA}$ & $1 \mathrm{M}$ & 0 & - \\
\hline $\mathrm{C} 2$ & $75-125+$ & 10YR 5/4 & A & $0 \mathrm{MA}$ & $1 \mathrm{M}$ & & - \\
\hline
\end{tabular}

Material original: Ceniza volcánica

\begin{tabular}{|c|c|c|c|c|c|c|c|c|}
\hline Oi & $0-4$ & - & - & - & - & - & - & - \\
\hline Oe & $4-5$ & - & - & - & - & - & - & - \\
\hline A & $5-17$ & $10 Y R 3 / 2$ & $\mathrm{AF}$ & 2 GR F & $3 \mathrm{M}$ & 0 & - & C S \\
\hline $\mathrm{AC}$ & $17-32$ & 10YR $3 / 3$ & FA & $1 \mathrm{SBK} F$ & $3 \mathrm{M}$ & 0 & - & $\mathrm{CS}$ \\
\hline $\mathrm{C} 1$ & $32-57$ & 10YR 3/3,5 & FA & 2 SBK M & $2 \mathrm{C}$ & 10 & - & $\mathrm{CS}$ \\
\hline $\mathrm{C} 2$ & $57-125+$ & 10YR 4/6 & FA & $2 \mathrm{SBK} M$ & $2 \mathrm{C}$ & 10 & - & - \\
\hline
\end{tabular}

a Color: Color según (according to) Munsell Soil Color Charts (1990).

b Textura (texture): $\mathrm{A}=$ arena (sand), $\mathrm{l}=$ limo (silt), a = arcilla (clay), $\mathrm{F}=$ franco (loam); $\mathrm{CNX}=$ extremadamente lajoso (extremely flagstony), PST $=$ con para-piedras (para-stony).

c Estructura (structure): $0=\sin$ estructura de agregados (structureless), $1=$ débil (weak), $2=$ moderada (moderate), $3=$ fuerte (strong); MA = masivo (massive), GR = granular (granular), SBK = bloques subangulares (subangular blocky); WEG = cuneiforme (wedge-shaped); $\mathrm{F}=$ fina (fine), $\mathrm{M}=$ media (medium), $\mathrm{CO}=$ gruesa (coarse), $\mathrm{VC}=$ muy gruesa (very coarse).

d Raíces (roots): en blanco (in black) $=$ ausentes (none), $1=$ pocas (few), $2=$ comunes (common), $3=$ abundantes (many); $\mathrm{F}=$ finas (fine), $\mathrm{M}=\mathrm{me}-$ dias (medium), $\mathrm{C}=$ gruesas (coarse), $\mathrm{VC}=$ muy gruesas (very coarse).

e Rasgos redoximórficos (redoximorphic features): en blanco (in black) $=$ ausentes (none), $\mathrm{f}=$ pocas (few), $\mathrm{c}=$ comunes $($ common), $\mathrm{m}=\mathrm{abundantes}$ (many); 1 = finos (fine), 2 = medios (medium), 3 = gruesos (coarse), $4=$ muy gruesos (very coarse); $\mathrm{D}=$ precisos (distinct), $\mathrm{P}=$ prominentes (prominent).

f Límite de horizontes (horizon boundary): $\mathrm{A}=$ abrupto (abrupt), $\mathrm{C}=$ claro (clear), $\mathrm{G}=$ gradual (gradual); $\mathrm{S}=$ plano (smooth), $\mathrm{W}=$ ondulado (wavy), I = irregular (irregular). 


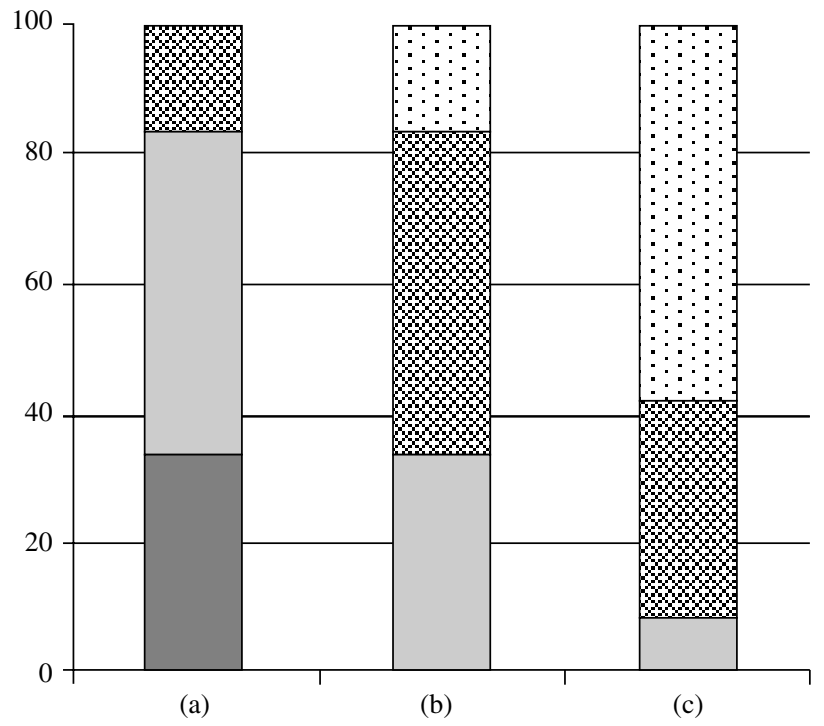

Tipos de estructura

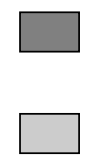

Depósito glacial de texturas finas / Fine glacial deposit

Depósito volcánico sobre material de textura fina / Volcanic deposit on fine material

Depósito volcánico sobre material grueso /

Volcanic deposit on coarse material

$\therefore$ Depósito aluvial / alluvial deposit

Figura 4. Materiales originarios del suelo en bosques de A. chilensis con distinto tipo de estructura. Se indica el porcentaje de parcelas de diferente material original para cada tipo de estructura.

Soil parent materials in A. chilensis forests corresponding to different stand structure types.

presentaron árboles remanentes de gran porte, los cuales frecuentemente evidenciaron cicatrices de fuego en la base del fuste.

Las parcelas con un rango acotado de DAP, donde dominaron los árboles de DAP $<15 \mathrm{~cm}$, se asociaron a suelos sin ceniza alofanizada, cuyo material original correspondió a un depósito glacial de textura fina, o a un depósito somero de ceniza depositado sobre material de texturas finas. Estos rodales tendieron a presentar drenajes deficientes y evidenciaron una baja $\mathrm{AB}$ y bajas alturas dominantes. El crecimiento en altura de los árboles dominantes es un indicador sensible de la calidad del sitio (Daniel et al. 1982, Donoso 1997). Las parcelas donde los árboles de DAP muy pequeño comenzaron a ser eliminados por competencia, y predominaron los árboles de hasta $35 \mathrm{~cm}$, se asociaron a suelos con ceniza volcánica alofanizada y evidenciaron mayor área basal y altura dominante que la estructura anterior. Las parcelas
Cuadro 4. Valores medios y error estándar de los parámetros dasométricos de parcelas asintomáticas y de parcelas afectadas por "mal del ciprés"

Mean and standard error of stand parameters of asymptomatic and symptomatic plots.

\begin{tabular}{ccc}
\hline Parámetro & $\begin{array}{c}\text { Parcelas } \\
\text { asintomáticas }\end{array}$ & $\begin{array}{c}\text { Parcelas } \\
\text { afectadas }\end{array}$ \\
\hline $\mathrm{N}^{\mathrm{o}}$ cipreses / ha ${ }^{\mathrm{ns}}$ & $2.152 \pm 283$ & $1.665 \pm 205$ \\
Área basal $\left(\mathrm{m}^{2} / \mathrm{ha}\right)^{\mathrm{ns}}$ & $75,9 \pm 7,4$ & $77,2 \pm 7,1$ \\
$\mathrm{DCM}(\mathrm{cm})^{\mathrm{ns}}$ & $23,6 \pm 2,1$ & $27,8 \pm 3,1$ \\
${\text { DAP dominante }(\mathrm{cm}){ }^{\mathrm{ns}}}^{\mathrm{n}}$ & $45,2 \pm 3,6$ & $52,1 \pm 5,2$ \\
${\text { H dominante }(\mathrm{m}){ }^{\mathrm{ns}}}^{\text {Número de anillos }}{ }^{\mathrm{ns}}$ & $18,2 \pm 1,0$ & $19,1 \pm 0,9$ \\
\hline
\end{tabular}

ns diferencias no significativas $(P>0,05)$.

donde se evidenciaron árboles remanentes de gran porte correspondieron, en su mayoría, a suelos aluviales con aporte de ceniza volcánica y a suelos volcánicos. Estos resultados evidencian la importancia del material original del suelo y del grado de alofanización de la ceniza volcánica como determinante del tipo de estructura, corroborando la hipótesis planteada. Sin embargo, la historia de disturbio también condiciona la estructura del bosque (Lorimer 1985). El 75\% de las parcelas con árboles de gran DAP, remanentes de antiguos incendios, se halló asociadó a cursos de agua (ríos y arroyos). Este hecho sugiere que se trata de sectores donde fue diferente el grado de disturbio generado por el fuego, dado que se localizan cercanos a cursos de agua, constituyendo áreas naturalmente protegidas (Kozlowski y Ahlgren 1974, Little 1974, Wright y Bailey 1982). Así, si bien los tipos de estructura hallados en bosques de A. chilensis coinciden con características edáficas particulares, también estarían fuertemente determinadas por el tipo y grado de disturbio generado por el fuego, como sucede en otras especies forestales (Lorimer 1985).

La declinación del bosque estuvo presente en los distintos tipos de estructura, evidenciando que la enfermedad no está restringida a una estructura en particular. En general, las parcelas asintomática y afectada de un mismo sitio de muestreo correspondieron al mismo tipo de estructura y no evidenciaron diferencias en altura dominante y área basal. De manera que no existió evidencia que valide la hipótesis planteada al respecto. La Manna y Rajchenberg (2004ab) identificaron fuertes diferencias de micrositio entre parcelas asintomáticas y afectadas por "mal del ciprés", estando el bosque enfermo asociado a sectores peor drenados que el bosque asintomático; sin embargo, esto no parece determinar diferencias significativas en la estructura del bosque.

Mueller-Dombois (1992) incluye la estructura del rodal como un componente de la susceptibilidad al decaimiento, indicando que las estructuras simplificadas, donde 
domina una cohorte de una única especie, desestabilizan el sistema forestal. El hecho de que las cuatro parcelas control correspondieran al tipo de estructura (c) (rodales con árboles remanentes de gran porte) sugiere que podría existir una diferente vulnerabilidad a la enfermedad en función de la estructura del bosque. En futuros estudios debería evaluarse la superficie afectada del rodal para cada tipo de estructura.

Independientemente del estado sanitario de los árboles, muchos de los tarugos para el conteo de anillos no pudieron obtenerse completos, producto de pudriciones presentes en el duramen de los árboles (Barroetaveña y Rajchenberg 1996). Esto determinó que se contara con escasos datos de edad exacta de los árboles. Por otra parte, los árboles enfermos presentan un crecimiento radial desigual en función de las raíces que presentan pudriciones, y puede ser diferente el número de anillos de acuerdo a la zona donde se obtenga el tarugo (Rajchenberg y Cwielong 1993). A pesar de estas limitantes, es interesante remarcar que edades de alrededor de 170 años se han registrado tanto en árboles de $55 \mathrm{~cm}$ de DAP, como en árboles de más de $80 \mathrm{~cm}$ de DAP, probablemente debido a la densidad o a la calidad de sitio (el primer caso correspondió a un suelo arcilloso, somero y de características vérticas con precipitaciones de $690 \mathrm{~mm}$ anuales; en tanto el de mayor DAP correspondió a un suelo aluvial con aporte de ceniza y $1.200 \mathrm{~mm}$ anuales de precipitación). La edad de muerte de los árboles en función del sitio es un tema a seguir analizando, que requiere de estudios dendrocronológicos exhaustivos.

\section{CONCLUSIONES}

Se evidenciaron tres tipos de estructura de bosque: (a) rodales con dominancia de árboles de DAP $<15 \mathrm{~cm}$, con bajos valores de área basal y altura dominante, asociados a suelos no volcánicos o volcánicos no alofanizados; (b) rodales con predominio de los árboles de 5 a $35 \mathrm{~cm}$ de DAP, con valores de área basal y altura dominante intermedios, asociados a suelos volcánicos alofanizados, y (c) rodales con predominio de los árboles de 5 a $45 \mathrm{~cm}$ de DAP y con árboles de gran porte sobrevivientes al fuego, con valores máximos de área basal y altura dominante, asociados a sectores aledaños a cursos de agua con suelos aluviales o volcánicos.

La declinación del bosque estuvo presente en los distintos tipos de estructura, evidenciando que la enfermedad no está restringida a una estructura en particular.

\section{AGRADECIMIENTOS}

Los autores desean agradecer al técnico forestal $\mathrm{Lu}-$ ciano Taladriz, a los ingenieros Carlos Buduba y Jorge Irisarri, por su asistencia en diversos aspectos del traba- jo, y a dos árbitros anónimos cuyas observaciones y sugerencias han permitido mejorar el manuscrito. Este estudio fue financiado por el Consejo Nacional de Investigaciones Científicas y Técnicas (CONICET) mediante el PIP 2166/00.

\section{REFERENCIAS}

Afifi AA, V Clark. 1984. Computer-Aided Multivariate Analysis. Belmont, USA. Lifetime Learning 3 Publications. $458 \mathrm{p}$.

Anchorena J, A Cingolani. 2002. Identifying habitat types in a disturbed area of the forest-steppe ecotone of Patagonia. Plant Ecology 158(1):97-112

Apcarian A, J Irisarri. 1993. Caracterización mineralógica de suelos desarrollados sobre cenizas volcánicas en las provincias de Neuquén y Río Negro (R.A.). Actas II Jornadas de Vulcanología, Medio ambiente y Defensa Civil. Zapala, Argentina. 23 p.

Baccalá N, P Rosso, M Havrylenko. 1998. Austrocedrus chilensis mortality in the Nahuel Huapi National Park (Argentina). Forest Ecology and Management 109:261-269.

Barroetaveña C, M Rajchenberg. 1996. Hongos Aphyllophorales (Basidiomycetes) que causan pudriciones en Austrocedrus chilensis en pie. Boletín de la Sociedad Argentina de Botánica 31:201-216.

Bava J, H Gonda. 1993. Propuesta silvícola preliminar de manejo para ciprés de la cordillera. Actas Congreso Forestal Argentino y Latinoamericano. Paraná, Argentina. $11 \mathrm{p}$.

Bran D, A Pérez, D Barrios, M Pastorino, J Ayesa. 2002. Ecoregión valdiviana: distribución actual de los bosques de "ciprés de la cordillera" (Austrocedrus chilensis) - Escala 1:250.000. Bariloche, Argentina. Instituto Nacional de Tecnología Agropecuaria, Administración de Parques Nacionales, Fundación Vida Silvestre. 12 p. (Informe preliminar).

Calí SG. 1996. Austrocedrus chilensis: estudio de los anillos de crecimiento y su relación con la dinámica del "Mal del Ciprés” en el P.N. Nahuel Huapi, Argentina. Tesis Licenciado en Ciencias Biológicas. Bariloche, Argentina. Centro Regional Universitario Bariloche, Universidad Nacional del Comahue. 143 p.

Colmet Dâage F, ML Lanciotti, A Marcolín. 1995. Importancia forestal de los suelos volcánicos de la Patagonia Norte y Central. Bariloche, Argentina. Instituto Nacional de Tecnología Agropecuaria. 27 p.

Daniel TW, JA Helms, FS Backer. 1982. Principios de silvicultura. México DF, México. McGraw-Hill. 492 p.

Davies BE. 1974. Loss-on ignition as an estimate of soil organic matter. Soil Science Proceedings 38:150.

Dezzotti A, L Sancholuz. 1991. Los bosques de Austrocedrus chilensis en Argentina: ubicación, estructura y crecimiento. Bosque 12(2):43-52.

Díaz-Vaz JE. 1985. Austrocedrus chilensis. Descripción anatómica. Bosque 6(1):49-50.

Donoso C. 1993. Bosques templados de Chile y Argentina. Variación, estructura y dinámica. Santiago, Chile. Editorial Universitaria. 484 p. 
Donoso C. 1997. Ecología Forestal: El bosque y su medio ambiente. $5^{\circ}$ ed. Santiago, Chile. Editorial Universitaria. $369 \mathrm{p}$.

Eswaran H, P Reichi, L Quandt. 1999. Vertisols: their properties, classification, distribution and management. Smith Memorial Slide Collection, USDA. Consultado 4 abril 2004. Disponible en: http://www.soils.usda.gov/use/worldsoils/vertisols/vertisols.pdf.

Fieldes M, KW Perrot. 1966. The nature of allophane in soils Part 3: rapid field and laboratory test for allophane. New Zealand Journal of Science 9:623-629.

Gobbi M, L Sancholuz. 1992. Regeneración postincendio del ciprés de la cordillera (Austrocedrus chilensis) en los primeros años. Bosque 13(2):25-32.

Goya JF, PF Yapura, JJ Ferrando, MD Barrera, MA Arturi. 1998. Crecimiento individual y de rodales de Austrocedrus chilensis en El Bolsón, Río Negro, Argentina. Revista de la Facultad de Agronomía (La Plata) 103:149-155.

Greslebin AG, L La Manna. 2004. El "mal del ciprés": una enfermedad de declinación de los bosques de Austrocedrus chilensis en Patagonia. Actas II Reunión Binacional de Ecología. Mendoza, Argentina. p. 258.

Greslebin AG, EM Hansen, L Winton, M Rajchenberg. 2004. "Mal del ciprés" (Austrocedrus chilensis decline) in Patagonia: are Phytophthora species involved? Proceedings 3rd Workshop of IUFRO Working Party 7.02.09 Phytophthora in forests and natural ecosystems. Freising, Alemania. p. 33.

Greslebin AG, EM Hansen, L. Winton, M Rajchenberg. 2005. Phytophthora species from declining Austrocedrus chilensis forests in Patagonia, Argentina. Mycologia 97:218-228.

Haller M. 2001. Descripción Geológica de la Hoja 4372-IV Trevelin, Chubut. Buenos Aires, Argentina. Servicio Geológico Minero. (Informe $\mathrm{N}^{\circ} 322$ ).

Havrylenko M, P Rosso, SB Fontenla. 1989. Austrocedrus chilensis: contribución al estudio de su mortalidad en Argentina. Bosque 10(1):29-36.

Hranilovic S. 1988. Informe histórico sobre el mal del ciprés de la cordillera (Austrocedrus chilensis). Revista de la Asociación Forestal Argentina 42:58-62.

Irisarri J. 2000. La propuesta de reclasificación de los Andepts de Argentina, de acuerdo al Orden Andisoles. Workshop Soil Taxonomy. Instituto Nacional de Tecnología Agropecuaria, AICET, Asociación Argentina de la Ciencia del Suelo. p. 18-27.

Kozlowsky TT, CE Ahlgren. 1974. Fire and Ecosystems. Nueva York, USA. Academic Press. 542 p.

La Manna L. 2005. Caracterización de los suelos bajo bosque de Austrocedrus chilensis a través de un gradiente climático y topográfico en Chubut, Argentina. Bosque 26(2):137-153.

La Manna L, F Carabelli. 2005. Distribución espacial de la declinación de los bosques de Austrocedrus chilensis en el Valle 16 de Octubre (Chubut, Argentina). Actas Primeras Jornadas Argentinas de Ecología de Paisajes. Buenos Aires, Argentina.

La Manna L, M Rajchenberg. 2004a. The decline of Austrocedrus chilensis forests in Patagonia, Argentina: soil features as predisposing factors. Forest Ecology and Management 190:345-357.
La Manna L, M Rajchenberg. 2004b. Soil properties and Austrocedrus chilensis decline in Central Patagonia, Argentina. Plant and Soil 263:29-41.

La Manna L, CG Buduba, J Irisarri, F Valenzuela. 2004. Vertisoles en los bosques de Austrocedrus chilensis. Revista Científica Agropecuaria 8:73-78.

Le-Quesne C. 1988. Caracterización de bosques de ciprés de la cordillera (Austrocedrus chilensis (D. Don) Florin et Boutelje), en Radal-7 tazas, Séptima Región, Chile. Tesis Ingeniero Forestal. Valdivia, Chile. Facultad de Ciencias Forestales, Universidad Austral de Chile.

Little S. 1974. Effects of fire on Temperate Forests: northeastern United States. In Koslowsky T, CE Ahlgren eds. Fire and Ecosystems. Nueva York, USA. Academic Press. p. $225-250$.

Loguercio G. 1997. Die Erhaltung der Baumart "Ciprés de la Cordillera", Austrocedrus chilensis (D. Don) Florin et Boutelje, durch nachaltige Nutzung. Tesis Doctor en Ciencias Forestales. Facultad Ludwig-Maximiliams, Universidad de München. Freising, Alemania. 212 p.

Loguercio G, P Burschel, M Rey. 1999. El bosque de Ciprés de la Cordillera: su conservación y uso. Esquel, Argentina. Centro Forestal CIEFAP. Folleto de divulgación $\mathrm{N}^{\circ}$ 14. $22 \mathrm{p}$

López CR. 1996. La carta de suelos en apoyo a la evaluación del potencial forestal de las tierras de la región andina patagónica norte. In Moscatelli G, J Panigatti, R Di Giacomo eds. Utilización de la cartografía para el uso sustentable de las tierras. Buenos Aires, Argentina. Instituto Nacional de Tecnología Agropecuaria.

Lorimer CG. 1985. Methodological considerations in the analysis of forest distrurbance history. Canadian Journal of Forest Research 15:200-213.

Manion PD. 1991. Tree disease concepts. Englewood Cliffs, USA. Prentice-Hall. $2^{\mathrm{a}}$ ed. 402 p.

Manion PD, D Lachance. 1992. Forest Decline Concepts. St. Paul, USA. The American Phytopathological Society Press. 249 p.

Marbán L. 2004. Capacidad de intercambio catiónico y cationes intercambiables. In Sistema de Apoyo Metodológico para Laboratorios de Análisis de Suelos y Aguas. Buenos Aires, Argentina. Secretaría de Agricultura Ganadería y Pesca.

Mueller-Dombois D. 1992. A natural dieback theory, cohort senescence as an alternative to the decline disease theory. In Manion PD, D Lachance eds. Forest Decline Concepts. St. Paul, USA. The American Phytopathological Society Press. p. 26-37.

Pritchett WL. 1986. Suelos Forestales: propiedades, conservación y mejoramiento. México DF, México. Editorial Limusa. $634 \mathrm{p}$.

Rabassa J, O Martínez, E Getar. 2000. Geomorfología del segmento de Cordillera Patagónica y Patagonia Extrandina entre $\operatorname{los} 43^{\circ}$ y $44^{\circ}$ Lat. Sur, Prov. de Chubut. Comodoro Rivadavia, Argentina. Universidad Nacional de la Patagonia S. J. Bosco, Secretaría de Ciencia y Técnica. 35 p. (Informe final PI $N^{\circ} 215$ ).

Raffaele E, M Gobbi. 1996. Seed bank composition and variability in Austrocedrus chilensis forest sites in Patagonia, Argentina. International Journal of Ecology and Environmental Sciences 22:59-72. 
Rajchenberg M, P Cwielong. 1993. El mal del ciprés (Austrocedrus chilensis): su relación con las pudriciones radiculares y el sitio. Actas Congreso Forestal Argentino y Latinoamericano, Paraná, Argentina.

Rajchenberg M, C Barroetaveña, P Cwielong, M Rossini, D Cabral, A Sívori. 1998. Fungal species associated with the decline of Austrocedrus chilensis in Patagonia, Argentina: preliminary results. In Delatour L, J Guillaumin, B Lung-Escarmant, B Marçais eds. Proceedings $9^{\text {th }}$ International Conference on Root \& Butt Rots, Carcans, Francia. Les Colloques 89:235-244.

Ramsey FL, DW Schafer. 1997. The Statistical Sleuth: a course in methods of data analysis. Belmond, USA. Duxbury Press. $742 \mathrm{p}$.

Relva MA, TT Veblen. 1998. Impacts of introduced large herbivores on Austrocedrus chilensis forests in northern Patagonia, Argentina. Forest Ecology and Management 108:27-40.

Rosso P, M Baccalá, M Havrylenko, S Fontenla. 1994. Spatial pattern of Austrocedrus chilensis wilting and the scope of autocorrelation analysis in natural forests. Forest Ecology and Management 67:273-279.

Schlatter J, V Gerding. 1995. Método de clasificación de sitios para la producción forestal, ejemplo en Chile. Bosque 16(2):13-20.

Schlegel F. 1962. Hallazgo de un bosque de cipreses cordilleranos en la provincia de Aconcagua. Boletín de la Universidad de Chile 32:43-46.

Schmaltz J, H Gonda. 1991. Descripción de un bosque nativo de ciprés (Austrocedrus chilensis) y propuestas para su futuro manejo. Actas II Congreso Internacional de Gestión en Recursos Naturales. Valdivia, Chile. II:497-505.

Schoeneberger PJ, DA Wysocky, E Benham, W Broderson. 1998. Field book for describing and sampling soils. Lin- coln, USA. Natural Resources Conservation Service, USDA, National Soil Survey Center. Traducción al castellano 2000. Libro de Campaña para descripción y muestreo de suelos, Instituto Nacional de Tecnología Agropecuaria. Buenos Aires, Argentina. 159 p.

Shaw JD, EC Packee, C Ping. 2001. Growth of balsam poplar and black cottonwood in Alaska in relation to landform and soil. Canadian Journal of Forest Research 3:1793-1804.

Sokal RR, FJ Rohlf. 1999. Introducción a la Bioestadística. México DC, México. Editorial Reverté. 362 p.

Urretavizcaya MF, GE Defossé. 2004. Soil seed bank of Austrocedrus chilensis (D. Don) Pic. Sern. et Bizarri related to different degrees of fire disturbance in two sites of southern Patagonia, Argentina. Forest Ecology and Management 187:361-372.

Veblen TT. 1992. Regeneration dynamics. In Glenn-Lewin, DC, RK Peet, TT Veblen eds. Plant Succession: theory and prediction. Londres, Reino Unido. Chapman \& Hall. p. 152-187.

Veblen T, FM Schlegel. 1982. Reseña ecológica de los bosques del sur de Chile. Bosque 4(2):73-115.

Veblen TT, BR Burns, T Kitzberger, A Lara, R Villalba. 1995. The ecology of the conifers of southern South America. In Enright NS, RS Hill eds. Ecology of the southern conifers. Melbourne, Australia. Melbourne University Press. p. 120-155.

Veblen TT, T Kitzberger, BR Burns, AJ Rebertus. 1996. Perturbaciones y dinámica de regeneración en bosques andinos del sur de Chile y Argentina. In Armesto JJ, C Villagrán, MK Arroyo eds. Ecología de los bosques nativos de Chile. Santiago, Chile. Editorial Universitaria. p. 169-198.

Wright HA, AW Bailey. 1982. Fire Ecology: United States and southern Canada. Nueva York, USA. John Wiley \& Sons. $501 \mathrm{p}$. 